\title{
The Meson Spectroscopy Program at the Jefferson Laboratory
}

\author{
A. Filippi ${ }^{1, a}$ for the CLAS Collaboration ${ }^{2, b}$ \\ ${ }^{1}$ I.N.F.N. sez. di Torino, via P. Giuria, 1, 10125 Torino, Italy \\ ${ }^{2}$ Thomas Jefferson National Accelerator Facility, 12000 Jefferson Avenue, Newport News VA 23606, U.S.A.
}

\begin{abstract}
The experimental techniques that will be applied by the next generation meson spectroscopy experiments at JLab are described. For the first time, these experiments will be able to exploit the features of a photon beam of unprecedented intensity and momentum resolution, that will allow to perform precision studies of meson states with masses below $3 \mathrm{GeV} / \mathrm{c}^{2}$. Photon induced reactions will enhance the production of spin-1 mesons, that are of particular interest according to the most recent Lattice QCD calculations of the lightest exotic hybrid meson.
\end{abstract}

\section{Introduction}

One of the still open questions in hadron physics is the origin of masses and the structure of the meson spectrum. Experimental observations have indeed pointed out that a constituent quark model (CQM) interpretation is not enough to account for all the hadron properties, but gluonic degrees of freedom have necessarily to be taken into account [1]. As a consequence of the non-abelian nature of the Quantum Chromodynamics field theory (QCD), self interactions between the gluons may occur, which could allow for the formation of extra states not foreseen by CQM. They are known as glueballs (composed by gluons only, ggg), hybrids (composed by a mixture of quarks and gluons, $\bar{q} q g$ ), and multiquark states (featuring a more complex structure, like, for instance, molecular-like $\bar{q} \bar{q} q q$ ). Some of these states could bear exotic quantum numbers, not allowed for "conventional" hadrons. In the last 50 years many searches have been pursued, exploiting different experimental techniques in production reactions induced by different probes, to extend the knowledge of the meson spectrum and prove the existence of such exotic states. Even though some of the features (like decay patterns) of some exotic states should help disentangling them from conventional mesons, their observation has not been conclusive so far, and none of the found candidates has actually been confirmed. This is also due to the fact that, especially in the light meson sector, many states overlap in the same mass region, and have similar and relatively large widths, on the order of $100 \mathrm{MeV}$. Therefore, their unambiguous extraction and observation is a difficult task. The situation is possibly a little simpler in the case of mesons composed by heavy quarks, whose widths are narrower so the overlap among different states is limited. However, also in this case many new observations of unexpected

\footnotetext{
ae-mail: filippi@to.infn.it

${ }^{\mathrm{b}}$ CLAS Collaboration website: http://www.jlab.org/Hall-B
}

states call for alternative interpretations of the full hadron spectrum.

In order to provide a correct identification of the still unknown states, high statistics observations are necessary, possibly in different reactions and in different decay channels. This started to be possible only in the Nineties. The experiments in that decade had been able to collect a good amount of data, however they didn't always agree on the observed properties of similar objects, that sometimes were even in open contradiction. New high statistics and high precision spectroscopy experiments are therefore still needed.

From the theoretical point of view, big advances have been made in the last years by QCD calculations on the lattice, which are now able to predict most of the conventional meson spectrum in good agreement with experimental findings [2]. These confirmations of course strengthen the confidence in their predictive power for the searches of new states. According to these calculations, the lightest hybrids and glueballs are predicted in the $1.4-3 \mathrm{GeV} / c^{2}$ mass range: namely, at $2 \mathrm{GeV} / c^{2}$ for the lightest $J^{P C}=0^{+-}$ state, and at $1.6 \mathrm{GeV} / c^{2}$ for the $1^{-+}$one. This is actually the mass region which will be investigated by the new experiments foreseen to operate at the upgraded CEBAF machine at JLab.

A few observations in this mass range for some structures with exotic quantum numbers have already been reported by old experiments, but with relatively small statistics. A $\pi_{1}(1400)$ object, with $J^{P C}=1^{-+}$, was claimed to have been observed at BNL by the E852 experiment [3], in the $\eta \pi$ decay channel in the $\pi^{-} p \rightarrow \pi^{-} \eta p$ and $\pi^{-} p \rightarrow \pi^{0} \eta n$ reactions at $18 \mathrm{GeV} / c$ incident pion momentum. The VES experiment found a much weaker signal at $37 \mathrm{GeV} / c$ incident momentum and did not confirm this result [4]. In $\bar{p}$ annihilations at rest on hydrogen and deuterium, the Crystal Barrel experiment at LEAR reported as well the observation of the $\pi_{1}(1400)$ decaying in $\eta \pi$, seen in the 
$\bar{p} p \rightarrow \pi^{0} \pi^{0} \eta$ and $\bar{p} n \rightarrow \pi^{-} \pi^{0} \eta$ reactions [5]: the $\pi \pi$ decays of $\sigma$ and $\rho$, and the contribution of the $a_{2}$ (1320) were found not to be enough to reproduce correctly the observed Dalitz Plot.

A second $1^{-+}$state at a larger mass (about 1600 $\mathrm{MeV} / c^{2}$ ), was observed by E852 in the $\eta^{\prime} \pi$ and $\rho \pi$ decay channels, in the $\pi^{-} p \rightarrow 2 \pi^{-} \pi^{+} p$ and $\pi^{-} p \rightarrow \eta^{\prime} \pi^{-} p$ peripheral productions at $18 \mathrm{GeV} / c$ [6]. Recently, a signature for a $1^{-+}$state in the same mass region was found by the COMPASS experiment at CERN-SPS [7], in the $\pi^{-} \mathrm{Pb} \rightarrow \pi^{+} \pi^{-} \pi^{-} \mathrm{Pb}^{\prime}$ reaction at $190 \mathrm{GeV} / c$ incident momentum. This is so far the highest statistic signature available for such a structure.

\section{Meson spectroscopy with photons at JLab}

As already mentioned, several reactions and beams have been used so far for meson spectroscopy searches: just to remind the most important, high-energy meson (mainly pion) and proton beams, exploiting the peripheral and central production mechanisms, antinucleon annihilation at rest and in flight, which convey the formation of a gluon-rich environment suitable for glueball production, and $e^{+} e^{-}$annihilation. The latter reaction has been studied extensively since the LEP era, and is a suitable environment where also $\gamma \gamma$ collisions can be measured, which provide quite useful information as they are a natural antiglueball filter. Starting in the Nineties the $e^{+} e^{-}$annihilation was fruitfully exploited for meson spectroscopy studies at flavor factories, with successful experiments like BaBar at PEPII and Belle at KEK, that operated at the $\Upsilon(n S)$ masses, BES (and its present upgrade) at BEPC, operating in the charmonium mass region, and KLOE at DAФNE, which operated at the $\phi$ energy. The $e^{+} e^{-}$annihilation differs from hadronic reactions for the fact that only $1^{--}$systems can be formed, so these reactions provide naturally a powerful quantum number selection.

On the other hand, the use of electromagnetic probes, and in particular of photons, in fixed target reactions was not used very extensively for meson spectroscopy purposes, due to the lack of sources of suitable intensity and momentum resolution. Nevertheless, electromagnetic reactions could deliver important complementary information. Let us remind that, first of all, electromagnetic processes can be exactly reproduced to a high level of precision through QED diagrams thanks to the smallness of the electromagnetic coupling, fact is prevented in the case of strong interactions. Moreover, photons can excite with larger probability the production of spin-1 mesons as compared to pion or kaon induced reactions, since in the latter case a spin-flip is required. Therefore, the production of vector hybrid mesons could occur in photoproduction reactions with a rate comparable to those of conventional vector mesons $[8,9]$. This feature applies not only to hybrids, but also to spin- $1 \bar{s} s$ excitations, whose production rate is suppressed, in general, as a consequence of OZI rule. The issues which prevented the use of electromagnetic probes in the past will be overcome by the new ex- periments at the upgraded CEBAF machine at JLab, which is presently under commissioning and will be operating in the next years.

\subsection{Experiments at CEBAF-12 GeV}

The CEBAF electron machine has been recently upgraded to reach electron momenta up to $12 \mathrm{GeV} / c$, about doubling the energy available in the first phase of operation of the facility. Its commissioning in the new configuration started in Fall 2014. Four experimental halls will host several fixed target experiments: for Hall A, B, C the maximum energy for the delivered electron beam will be 10.9 $\mathrm{GeV}$, while the newly built Hall $\mathrm{D}$ will benefit of a maximum energy beam of $12 \mathrm{GeV}$. Details on the machine's expected performance may be found elsewhere [10]. As figures of merit, the beam power will reach $1 \mathrm{MW}$ with a current of $90 \mu \mathrm{A}$ maximum intensity, corresponding to a luminosity on fixed targets of about $10^{35} \mathrm{~cm}^{-2} \mathrm{~s}^{-1}$.

Two main experiments will be dedicated to a meson spectroscopy search program: GlueX in Hall D, and CLAS12 in Hall B. Their main features and their complementarity will be briefly described in the following.

\subsubsection{The GlueX Experiment}

The GlueX Experiment is a $4 \pi$ full acceptance spectrometer which will be completely dedicated to meson spectroscopy studies with a real photon beam. The photon beam will be produced by a dedicated facility based on electron bremsstrahlung on a diamond crystal, exploiting a technique well known and consolidated in experiments of the $6 \mathrm{GeV}$ CEBAF era. The obtained photon beam will have an energy in the $6-9 \mathrm{GeV}$ range with a 10 $\mathrm{MeV}$ resolution, and will have a linear polarization (measured collectively) from 15 to $50 \%$. Information on the photon beam will be obtained through a proper tagging equipment. The use of polarized photons is important to reduce the number of degrees of freedom and simplify the data analysis through partial-wave decomposition, whose number of components can thus be reduced, as will be described in the following. The photon flux will have an intensity of $10^{8}-10^{9} \mathrm{\gamma} / \mathrm{s}$, enough to succesfully perform high statistics spectroscopy studies.

A detailed description of the experimental apparatus may be found in Ref. [11]. Here it is enough to mention that the apparatus has been devised to feature a good hermeticity and acceptance. It includes particle identification detectors for the detection of kaons and strange hadrons, to study strangeness photoproduction, a topic still largely unknown, but features a limited momentum resolution, though enough for spectroscopy investigations. A sketch of the experimental apparatus is shown in Fig. 1.

\subsubsection{The CLAS12 Experiment and the Forward Tagger equipment}

The CLAS12 spectrometer is a multipurpose facility dedicated to hadron physics studies, from nuclear properties 


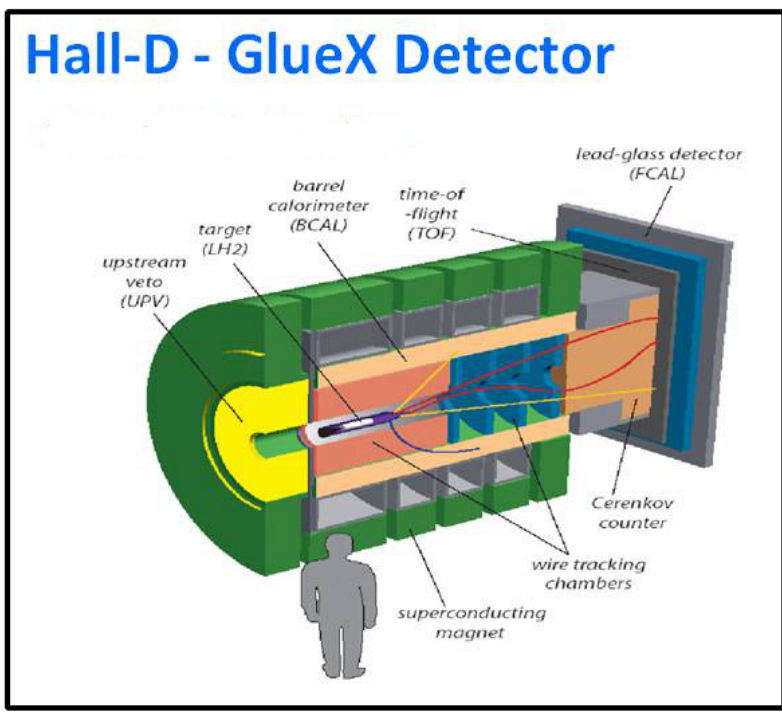

Figure 1. Schematic view of the GlueX experiment.

and structure to meson spectroscopy investigations. It is an upgraded version of the CLAS Experiment which operated until 2010 [12]. Due to the increased beam energy, as compared to the previous installation, the CLAS12 spectrometer has a more compact structure, which limits its geometric acceptance, although allowing an acceptable hermeticity. However, its detectors feature better momentum resolution and particle identification capabilities as compared to GlueX, therefore the two experimental setups offer complementary qualities.

A full description of CLAS12 is given elsewhere [13]. Two main sections constitute it, a forward part covering the $5^{\circ}-35^{\circ}$ angular region and a central one, covering polar angles up to $135^{\circ}$. In the forward part the detectors are arranged around the beam pipe in a six-fold symmetry, and form six identical spectrometers; in the central part they are arranged in cylindrical symmetry. In the following a list of the elements composing CLAS12 in its base configuration is given.

\section{The Forward Detector is composed of:}

- a torus magnet, composed of six kidney-shaped coils providing a toroidal magnetic field with a maximum intensity of $3.58 \mathrm{~T}$;

- a forward Micromegas tracker, composed by three layers, providing a spatial resolution of about $144 \mu \mathrm{m}$ in $x$ and $y$;

- a high threshold gas Cherenkov counter (HTCC);

- a drift chamber system, that together with the Micromegas tracker forms the Forward Tracking system. The Forward Tracker features a momentum resolution dependent on the polar angle and ranging from $0.3 \%$ at $5^{\circ}$ to $1 \%$ at $30^{\circ}$. The drift chambers consist of three super-layers (composed each of two drift planes staggered by $\pm 6^{\circ}$ ), one located in front of the torus coils outside the field region, the second between the coils where the field is maximum and the third outside them;
- a low threshold gas Cherenkov counter (LTCC);

- a Time-Of-Flight (TOF) system, composed by scintillators, able to provide a $\pi / K$ separation up to $3 \mathrm{GeV} / c$, with a time resolution depending on the polar angle and varying from 60 to $150 \mathrm{ps}$. Each of the six sectors is made of three sets of overlapping counters, forming panels of two types, that cover two different angular regions;

- a preshower calorimeter (PCAL), formed by 15 layers of plastic scintillator and lead, staggered by $120^{\circ} \mathrm{ev}$ ery layer pair; it is necessary to degrade the energy of the electromagnetic showers with momenta larger than $5 \mathrm{GeV} / c$ and to reduce the $\pi^{0}$ energy, to allow the following electromagnetic calorimeter to fully absorb the shower energy and to resolve the $\gamma$ 's from the $\pi^{0}$ decay with enough resolution;

- an electromagnetic calorimeter (EC), used to measure and identify electrons and photons with energy larger than $500 \mathrm{MeV}$ and $200 \mathrm{MeV}$, respectively, and provide their separation. The full PCAL+EC assembly will feature an energy resolution $\sigma_{E} / E \sim 0.1 / \sqrt{E}$;

The Central Detector is composed of:

- a superconducting solenoidal magnet, of cylindric geometry, providing a field of $5 \mathrm{~T}$. Besides from bending particles, it is also necessary to shield the Forward Detector from Møller electrons;

- the target system, located at the center; according to the experimental program, it can also include a longitudinally polarized cryogenic target;

- a barrel tracker, composed of three layers of Micromegas sandwiching two layers of double-sided silicon microstrip detectors, providing a momentum resolution of $5 \%$ at $1 \mathrm{GeV} / c$;

- a central TOF system (CTOF), used for particle identification through speed measurement, able to allow a $4 \sigma$ separation of pions from kaons up to $640 \mathrm{MeV} / c$. The detector is composed by 50 plastic scintillator counters, arranged as staves of $\mathrm{a} \sim 25 \mathrm{~cm}$ radius barrel inserted inside the solenoid;

- a central neutron detector, whose structure is similar to CTOF and is made by four cylindrical layers composed by 30 thick scintillators each. Its momentum resolution is about $5 \%$ and the neutron detection efficiency $\sim 10 \%$.

Other additional detectors will be added to the base configuration according to the purpose of the running experiment. A sketch of CLAS12 is shown in Fig. 2.

While GlueX will use a real photon bremsstrahlung beam, this will not be possible for CLAS12 as the bending dipole magnet available in Hall B will not be powerful enough to steer the $11 \mathrm{GeV}$ electron beam into the tagger beam dump. A new technique was therefore devised to produce a photon beam for the study of photoproduction reactions, based on electron scattering at very low transferred momentum $\left(Q^{2}<10^{-1}(\mathrm{GeV} / c)^{2}\right)$. In this situation the electrons are scattered at very small polar angles, and the reaction on protons is induced by quasi-real photons. A dedicated part of the CLAS12 apparatus, the 


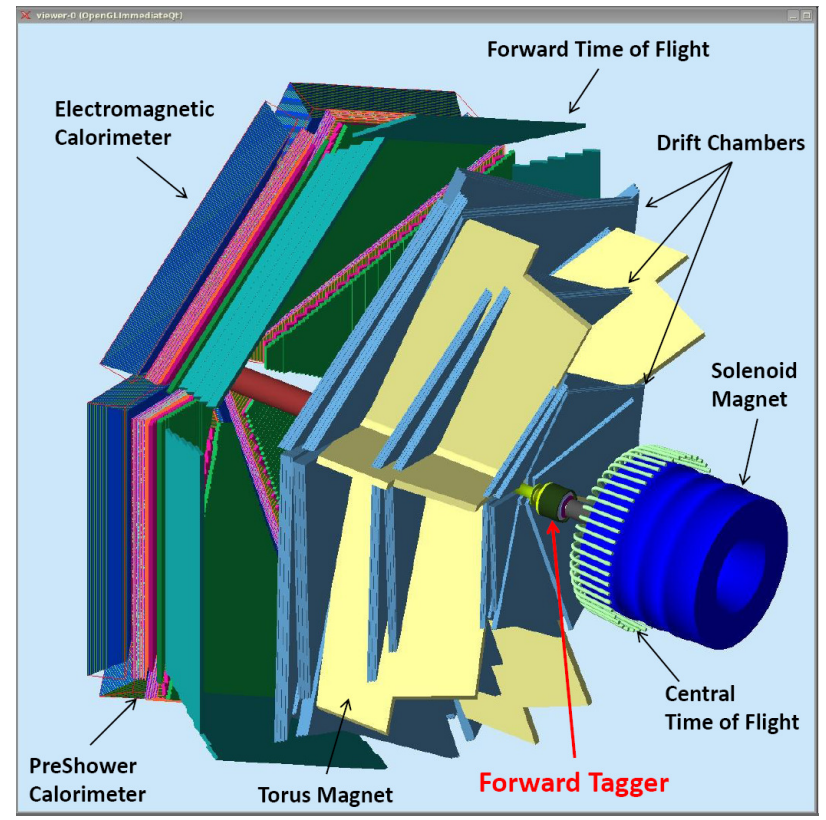

Figure 2. Schematic view of the CLAS12 experiment (exploded; beam comes from the right.)

Forward Tagger, was devised to detect these forward scattered electrons. For momentum transfers in the interval $0.01<Q^{2}<0.3(\mathrm{GeV} / \mathrm{c})^{2}$, the electrons scattered between $2.5^{\circ}$ and $4.5^{\circ}$ in polar angle in the lab have an energy in the $0.5-4.5 \mathrm{GeV}$ range, being produced by the interaction of $6.5-10.5 \mathrm{GeV}$ quasi-real photons on protons. Measuring the electron momentum, each virtual photon can be tagged. The use of the CLAS12 spectrometer to measure in coincidence the particles produced in the photoproduction reaction will allow to perform a complete event recontruction, necessary for meson spectroscopy studies. With this tagging technique one can measure also the virtual photon polarization, that is linear and can be deduced, event by event, from the energy and the angle of the scattered electron. The systematic uncertainty affecting the polarization depends only on the electron momentum resolution. High electron currents may be used, therefore a good luminosity can be obtained even with thin targets, that are not operable with real photon bremsstrahlung beams. For instance, using a $5 \mathrm{~cm}$ long $\mathrm{LH}_{2}$ target, the resulting hadronic rate will be equivalent to that achievable by a real photon flux of about $5 \times 10^{8} \gamma / \mathrm{s}$.

The Forward Tagger equipment, described in detail in Ref. [14], is located between the HTCC and the torus support, about $190 \mathrm{~cm}$ away from the target and fits within a $5^{\circ}$ cone around the beam axis. It is made up of:

- an electromagnetic calorimeter (FT-Cal): composed by $332 \mathrm{PbWO}_{4}$ crystals, $20 \mathrm{~cm}$ long and with square $15 \times 15$ $\mathrm{mm}^{2}$ cross-section. It is used to identify the scattered electron and measure its energy, from which the photon energy and its polarization can be deduced (the polarization being given by $\epsilon^{-1} \sim 1+v^{2} /\left(E E^{\prime}\right)$, where $v=E-E^{\prime}$ is the photon energy, and $E$ and $E^{\prime}$ the energies of the incident and of the scattered electron, respectively). It is also used to provide a fast trigger signal. Its expected design resolution is $\sigma_{E} / E \sim(2 \% / \sqrt{E(\mathrm{GeV})} \oplus 1 \%)$;

- a scintillator hodoscope made of plastic scintillator tiles: located in front of the calorimeter, is used to veto photons; its spatial and timing resolution is required to be comparable with FT-Cal's;

- a tracker: located in front of the hodoscope and composed by Micromegas detectors, is used to measure the angle of the scattered electron and the photon polarization plane.

A sketch of the Forward Tagger region is shown in Fig. 3.

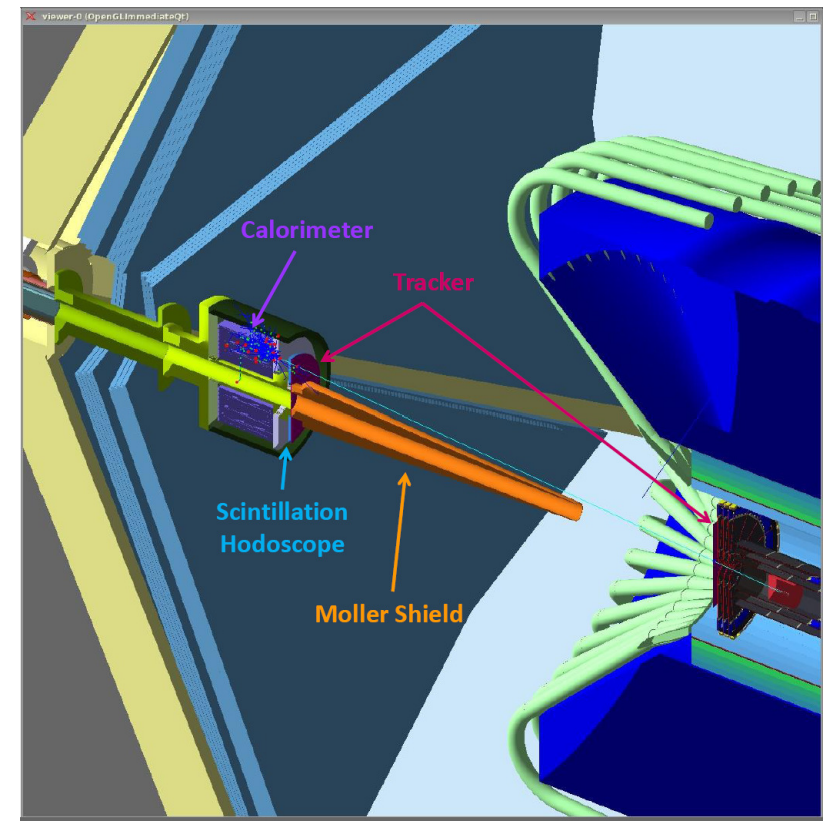

Figure 3. Schematic view of the Forward Tagger equipment, to be hosted in CLAS12.

\section{The MesonEX experiment at CLAS12}

The MesonEX experiment at CLAS12 (Exp-11-005 [15]) has as main goal the study of the meson spectrum in the $1-3 \mathrm{GeV} / \mathrm{c}^{2}$ mass range through quasi-real photon induced reactions, for the identification of gluonic excitations of mesons and other exotic quark configurations beyond CQM. The use of the Forward Tagger will allow to identify the photoproduction reaction on protons through the tagging of the forward scattered electron, while the full CLAS12 apparatus will perform a complete reconstruction and identification of the charged and neutral particles produced in the interaction. Some golden channels have been selected as particularly suitable for the search of exotic or still unknown particles, whose production, as mentioned before, will be favored in photoproduction especially in the case of spin-1 particles. In particular, the following reactions will potentially bring new useful information: 
$1^{-}$hybrids and exotics:

- $\gamma p \rightarrow 3 \pi N$

- $\gamma p \rightarrow \eta \pi^{0} p[16]$

- $\gamma p \rightarrow \eta \pi^{+} \pi^{-} p$

Hybrids with open strangeness and strangeonia:

- $\gamma p \rightarrow \phi \pi^{0} p$

- $\gamma p \rightarrow \phi \eta p$

- $\gamma p \rightarrow K \bar{K} \pi p$

Scalar mesons:

- $\gamma p \rightarrow \pi^{+} \pi^{-} p$

- $\gamma p \rightarrow K \bar{K} p$

As mentioned above, the study of the $3 \pi$ and $\eta \pi$ intermediate states will bring additional information to the recent observation by COMPASS of an isovector state in the $1400 \mathrm{MeV} / \mathrm{c}^{2}$ region [7].

Concerning the photoproduction of open and hidden strangeness, data on these reactions are missing as they were never studied thoroughly in the past due to the small production rates. In fact, the production of strange quarks in a non-strange environment involves disconnected quark diagrams whose occurrence is suppressed as a consequence of the OZI rule. Apart form a handful confirmed states, little is known for instance about the radial excitations of the $\phi(1020)$ meson, and even less about strangeonia with quantum numbers other than $1^{--}$. Smoking guns for the existence of such states would by their observations in the $\phi \eta$ or $\phi \pi$ invariant mass systems [8]; especially in the first case, due to the strange content of the $\eta$ meson, the production of strangeness should be eased [9].

Kaonia and open strangeness states can be produced in photoproduction reactions in this mass region as well. These states were widely studied in the past to disentangle between their production and that of $\eta$ excitations, which could possibly carry exotic quantum numbers. Many observation of $\eta$ 's and $f_{1}$ mesons have been reported since the Sixties, when the issue of the overlap of many axial and pseudoscalar states and the difficulty of their identification posed the so-called $E / \iota$ puzzle [17]. While annihilation experiments, and in particular OBELIX, could provide a solution to this puzzle addressing the production of several pseudoscalar and axial states, high statistics photoproduction reactions are expected to provide new complementary information which will be able to improve the knowledge in this sector.

In spite of the efforts by the experiments in the early Nineties, in particular those studying antinucleon annihilations, like Crystal Barrel and OBELIX, also in the scalar meson sector the situation is not completely clear yet. Observations have been made of several mesons whose existence was not foreseen by the CQM; one of them, the $f_{0}(1500)$, seemed to have the right features as lightest scalar glueball [18]. Among these, the most important is the fact that it was observed to decay in several channels, a clear hint to its flavor-blindness. However, more data are still desirable to confirm these properties and identify the other structures tentatively identified as scalars. All the existing observations make the interpretation of the scalar sector difficult, since it is populated, more than expected, by broad and overlapping states. In this mass region one should also recall the existence of the $\sigma$ state (also known as $f_{0}(500)$ ), corresponding to a very broad $\pi \pi$ non-resonant iso-scalar $S$-wave interaction, whose nature and properties are still unclear. Also scarcely known are the features of the $\kappa$, the analogous of $\sigma$ observed in the $K \bar{K}$ channel [19].

The MesonEX experiment is expected to collect data for 80 days at the full CEBAF luminosity ( $10^{35} \mathrm{~s}^{-1} \mathrm{~cm}^{-2}$ ), in parallel with other CLAS12 experiments studying electron interactions, but that will benefit of the presence of the Forward Tagger. The total expected trigger rate for photoproduction reactions is less than $10 \mathrm{kHz}$. In this data taking time, assuming an apparatus acceptance for four track events of the order of $15 \%$, it will be possible to collect as many as 3000 events per $10 \mathrm{MeV} / \mathrm{c}^{2}$ mass bin, for reactions with a cross section as small as 10 $\mathrm{nb}$, expected for instance for strangeonia production. This statistics is considered to be enough to perform detailed partial-wave analysis studies, as will be described in the following section.

\section{Data analysis: methods}

The extraction of signatures of meson production in photon induced reactions is performed through the widely known Partial Wave Analysis (PWA) method, by which the production cross sections are parameterized via the sum of partial amplitudes. Each amplitude enters in the sum with a weight, usually expressed as a complex number (including this way a phase which accounts for a coherent overlap), which is to be determined by fitting the total production amplitude to the data sample.

The partial amplitudes are function of several quantum numbers, both related to the initial and to the final state, assuming their conservation in a strong interaction process: the total spin $J$, the spatial parity $P$, the chargeconjugation $C$ and the total isospin $I$ (and the related $G$ parity), together with the relative angular momentum $L$ between the reaction products in the final state. The amplitudes, moreover, are dynamical functions of the produced particle momenta. For the description of each partial wave models have to be invoked which necessarily call for a certain degree of approximation for the description of the actual process. So far, the isobar model has been the most widely used, in which the final state is assumed to be reached through a series of two-body interactions [20]. This is a reasonable approximation, but neglects, for instance, possible many-body interactions in the intermediate steps. Moreover, it is prone to some flaws, like unitarity violations that can accidentally occur at certain stages of the process description. Usually isobar models have to be completed including the treatment of coupled channels. Coupled-channel data can be included in the global fits providing further constraints to the amplitude parameters; however, in several cases they are not available, so parameterizations from other experiments must be used and this is 
for instance a potential source of unitarity break-down. An alternative approach, mathematically preserving unitarity and causality, is based on dispersion relations and duality [21]. In this case the implementation, however, is not always straightforward and so far proved to be successful only in some simple cases, namely few-body decay amplitudes. However, quite recently some efforts have been spent in this direction, as this approach provides a more comprehensive and complete description of scattering and decay amplitudes [22].

Therefore, when trying to reproduce the data by means of these descriptions, one has to face a twofold problem: a) using a reliable and as accurate as possible model to reproduce the data in the best way and b) address the existence of additional effects, not included in the amplitude, to account for the possible remaining discrepancies between the data and the model. Signatures of exotic states are expected to show up as tiny effects, so the identification of such structures is a particularly delicate task. Care must also be taken, from the experimental side, to provide accurate acceptance corrections to the data, in order not to misidentify distortions caused by acceptance flaws or finite detector resolution as real physical effects.

Ideally, the most effective way to obtain a reliable description of the production of a given state would be to use as many experimental data samples as possible, obtained in different reactions and in different experimental environments; each will allow to impose additional constraints to the number of degrees of freedom, i.e. of free parameters to be determined by the fit. For this reason it is also important to exploit as much as possible the experimental conditions in which the reactions occur, upon which quantum numbers may vary. For instance, one method is to select the polarization of the beam, which defines the helicity of the initial state. A piece of information often exploited in $\bar{p}$ annihilation reactions is the density of the target (liquid or gaseous at different pressures): this is an important parameter to determine the partial wave in which the beam-target interaction occurs, as a consequence of the Stark effect.

The crossed information on the same objects obtained in different reactions is indeed one of the best handles to unambiguously identify new structures and pin down their properties.

\section{Data analysis: feasibility tests on CLAS12 data and performances}

In order to perform a reliable partial-wave analysis a good number of selected events, on the order of at least a few thousand per mass or momentum transfer bins, are necessary. Studies with Monte Carlo data have been made to check the capability of CLAS12 to face the mentioned problems and provide a good enough data sample to extract reliable information on new physical effects. To this purpose, some benchmark reactions have been investigated, in which new signals in known conditions have been injected (i.e., with known spin and fixed production rates), to check the reproducibility of the simulated spec- trum against acceptance distortions and an approximate amplitude description.

An example of a tested reaction is $\gamma p \rightarrow \pi^{+} \pi^{+} \pi^{-} n$, in which a sum of eight isobar channels was considered, corresponding to the known resonant states in $S-, P-, D-$, $F$-waves decaying in $\rho \pi$ or $f_{2}(1270) \pi$, plus an exotic signal in $P$-wave (standing for the $\pi_{1}(1600) 1^{-+}$state in its $\rho \pi$ decay). The simulated data were folded with the CLAS12 acceptance, processed through the full reconstruction chain, and then fitted via a proper partial-wave set in the framework of the isobar model and in selected bins of transferred momentum $t$. The obtained results, in two $t$ bins marked by points of different colors, are shown in Fig. 4. All known resonances $-a_{2}(1320)$ in $D$-wave, $a_{1}(1260)$ in $D$-wave, $\pi_{2}(1670)$ in the two decay channels $\rho \pi(P$ - and $F$-wave) and $f_{2} \pi$ ( $S$ - and $D$-wave) - are correctly reproduced at the right mass values, as well as the additional exotic signal. Its injected weight, at the level of $2 \%$ (corresponding to a production cross section of about $200 \mathrm{nb}$ ), was reproduced correctly as well. Similar tests (see for instance Ref. [16] for the $\gamma p \rightarrow \eta \pi^{0} p$ channel) show that partial-wave analyses will be feasible with CLAS12 data without any particular problem related to the limited apparatus acceptance, with a sensitivity on signal production which can reach the level of a few percent.

\section{Outlook and conclusions}

Nowadays many communities are involved all over the world in hadron spectroscopy studies, both from experimental and from the theoretical point of view. Experimental facilities are presently delivering, and will deliver in the near future, large amounts of good quality data that will allow to extract for the first time new information on many still unsolved issues from different reactions and experimental environments.

The required effort to analyse and interpret the data, especially in a comprehensive perspective, is huge, and the development of common tools and collective collaborative resources is mandatory to achieve this goal. This of course implies not only the development of a common analysis framework for data handling and interpretation through innovative and general-purpose partial-wave decomposition formalisms, but also requires the deployment of new computing standards for a most efficient and fast data access, distribution and processing.

Common tools to perform partial-wave analyses with standardized procedures, such as AmpTools [23] or ComPWA [24] have been developed in the latest years and are now freely available to the community. Concerning information technology infrastructures, Cloud computing looks as one the most suitable resources to effectively perform data distribution and mining among several experiments and laboratories. Concerning computational techniques, promising steps have recently been made in the study and test of GPU performances, for application to data processing and high level analyses.

In parallel, several groups have organized their efforts both to elaborate tools and provide data-analysis patterns 

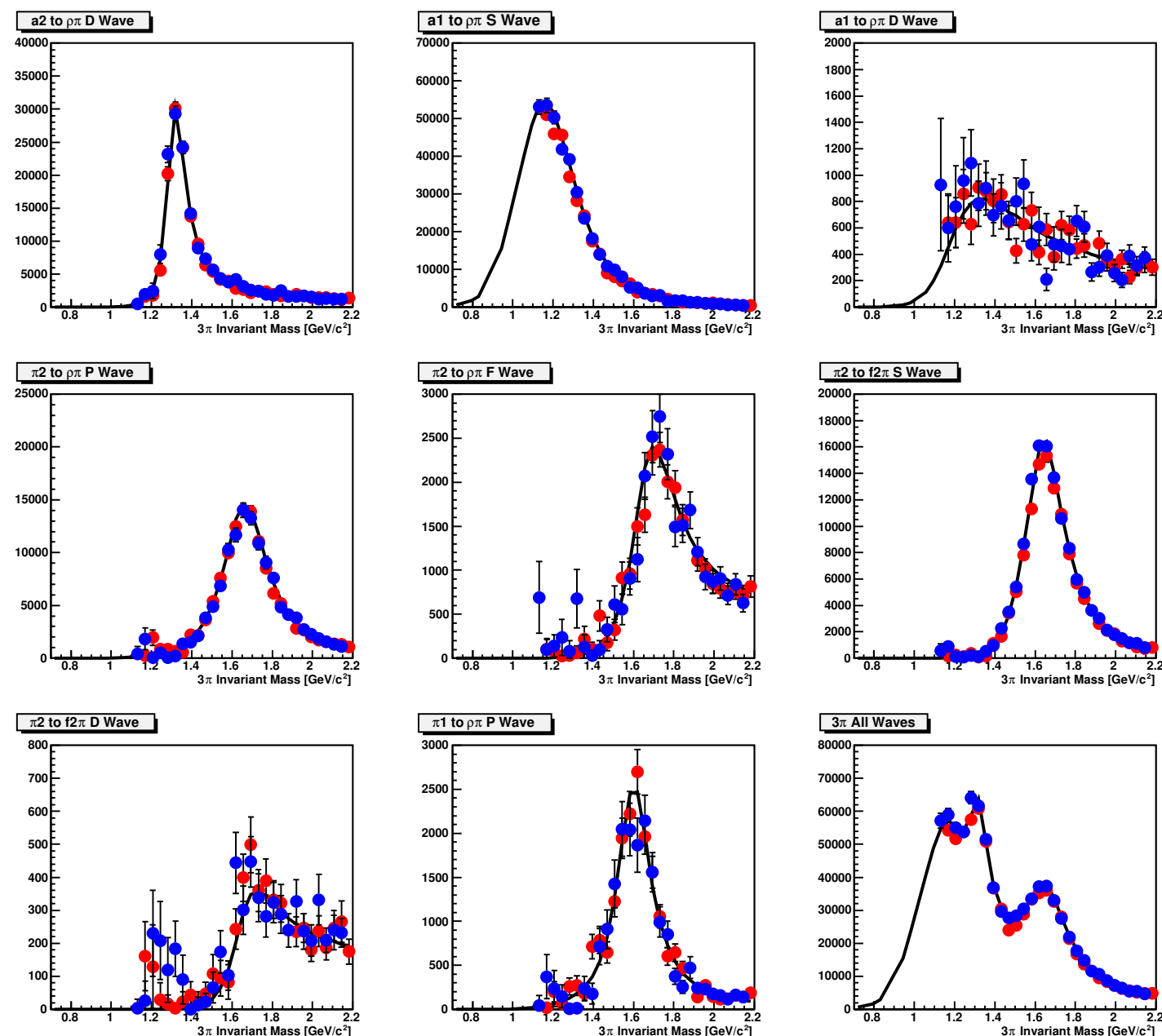

Figure 4. Intensities of eight isobar channels in the $\gamma p \rightarrow \pi^{+} \pi^{+} \pi^{-} n$ channel. In the bottom row, the last pad on the right corresponds to the total $3 \pi$ intensity, while the pad in the center correspond to the exotic signal injected in the pseudo-data sample. The black lines are the generated partial waves, while the points are the fit results for pseudo-data selected in the two bins centered at $t=0.2(\mathrm{GeV} / c)^{2}$ $\left(0.25(\mathrm{MeV} / c)^{2}\right.$ wide, blue points) and $t=0.5(\mathrm{GeV} / c)^{2}\left(1(\mathrm{MeV} / c)^{2}\right.$ wide, red points).

useful to the whole spectroscopy community. One of them, dedicated to the study of the photoproduction data from CLAS12 (and previous CLAS as well, used as benchmarks to test feasibility and explore new investigation grounds), is the Hadron Spectroscopy Center (HaSpect), based in Genova, Italy [25]. Experimentalists as well as theoreticians belonging to this group work in synergy to provide tools and patterns applied directly to the photoproduction case but extendable to other reactions as well.

Photoproduction experiments at JLab will play a big role in the coming future. At JLab, GlueX and CLAS12 will provide data samples of unprecedented quality and richness. Their contribution is expected to grow to sizeable relevance and have a big impact in the present and future meson spectroscopy experimental scenario, complementing the information that will be provided by $e^{+} e^{-}$ collisions (by BESIII and BelleII), by pion induced interactions (by COMPASS), by proton-proton interactions (at fixed target by LHCb, and in high-energy $p p$ collisions by ATLAS and CMS) and by antiproton-proton annihilations (by PANDA at FAIR).

\section{References}

[1] K.A. Olive et al. (Particle Data Group), Chin. Phys. C38, 090001 (2014); see for instance the number of observed states in the listing of light unflavored mesons: many more states than predicted by the simple CQM are reported.

[2] J. Dudek et al.,, Phys. Rev. D82 (2010), 034508; J. Dudek et al.,, Phys. Rev. D83 (2011), 111502

[3] E852 Collaboration, G.S. Adams et al., Phys. Rev. Lett. 81 (1998), 5760

[4] VES Collaboration, G.M. Beladidze, Phys. Lett. B213 (1993), 276

[5] Crystal Barrel Collaboration, A. Abele et al., Phys. Lett. B423 (1998), 175;

Crystal Barrel Collaboration, A. Abele et al., Phys. Lett. B446 (1998), 349

[6] E852 Collaboration, S.U. Chung et al. Phys. Rev. D65 (2002), 072001;

E852 Collaboration, E. I. Ivanov et al. Phys. Rev. Lett. 86 (2001), 3977; 
E852 Collaboration, M. Lu et al., Phys. Rev. Lett. 94 (2005), 032002

[7] COMPASS Collaboration, A. Alekseev et al., Phys. Rev. Lett. 104 (2010), 241803; COMPASS Collaboration, B. Grube et al., arXiv:1002.1272 [hep-ex] (2010)

[8] F. Close and P. Page, Phys. Rev. D52 (1995), 1706; A. Afanasev and P. Page, Phys. Rev. D57 (1998), 6771; A. Szczepaniak and M. Swat, Phys. Lett. B516 (2001), 72

[9] T. Barnes et al., Phys. Rev. D55 (1997), 4157;

T. Barnes, N. Black and P. Page, Phys. Rev. D68 (2003), 054014

[10] C.W. Leemann et al., Ann. Rev. Nucl. Part. Sci. 51 (2001), 413

[11] GlueX Collaboration, Mapping the Spectrum of Light Mesons and Gluonic Excitations with Linearly Polarized Photons, Proposal to Jefferson Laboratory PAC30, 2006; url: http://argus.phys.uregina.ca/cgibin/public/DocDB/ShowDocument?docid=1226

[12] B.A. Mecking et al., Nucl. Instr. Meth. A503 (2003), 513

[13] CLAS Collaboration, CLAS12 Technical Design Report, Tech. Rep. Jefferson Laboratory, 2008

[14] CLAS Collaboration, CLAS12 Forward Tagger (FT) Technical Design Report, Tech. Rep. Jefferson Lab, 2012; url: http://clasweb.jlab.org/wiki/index.php/ CLAS12_Technical_Design_Report

[15] CLAS Collaboration, Meson Spectroscopy with low $Q^{2}$ electron scattering in CLAS12, Proposal to Jefferson Laboratory PAC37, 2011
[16] A. Celentano, The Forward Tagger detectors for CLAS12 at Jefferson Laboratory and the MesonEx experiment, PhD Thesis, Università degli Studi di Genova (2014)

[17] A. Masoni, C. Cicalò and G.L. Usai, J. Phys. G32 (2006), R293

[18] Crystal Barrel Collaboration, C. Amsler et al., Phys. Lett. B342 (1995), 433;

Crystal Barrel Collaboration, C. Amsler et al., Phys. Lett. B353 (1995), 571;

Crystal Barrel Collaboration, C. Amsler et al., Phys. Lett. B385 (1996), 425;

OBELIX Collaboration, A. Bertin et al., Phys. Lett. B408 (1997), 476;

OBELIX Collaboration, A. Bertin et al., Phys. Rev D57 (1998), 55;

OBELIX Collaboration, M. Bargiotti et al., Phys. Lett. B561 (2003), 233

[19] J.R. Pelaez, These Proceedings

[20] K.M. Watson, Phys. Rev 88 (1952), 1163

[21] G. Veneziano, Nuovo Cim. 57 (1968), 180; R.C. Brower, Phys. Rep. 14 (1974); G. Altarelli, Phys. Rev. 183 (1969), 1469

[22] A. Szczepaniak and M. Pennington, Phys. Lett. B737 (2014), 283

[23] For AmpTools documentation, see for instance: http://amptools/sourgeforge.net/index.php/Main_Page

[24] For ComPWA documentation, see for instance: http://compwa.gitub.io/ComPWA

[25] E. Santopinto et al., J. Phys. Conf. Series 527 (2014), 012028 